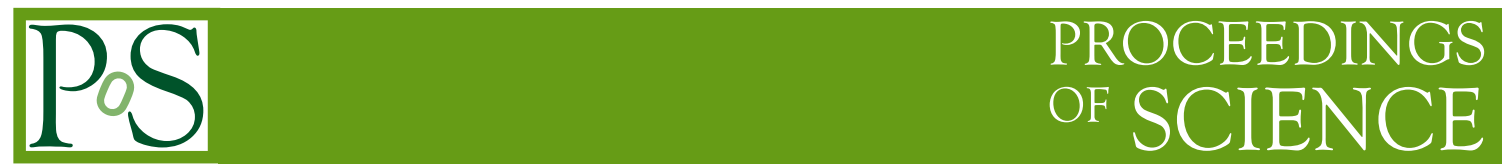

\title{
Electric Dipole Moment of the Neutron
}

\section{Eigo Shintani*}

RIKEN-BNL Research Center, Brookhaven National Laboratory, Upton, NY 11973, USA

E-mail: shintani@riken.jp

\section{Thomas Blum}

Physics Department, University of Connecticut, Storrs, CT 06269-3046, USA

E-mail: tolum@phys.uconn.edu

\section{Taku Izubuchi}

RIKEN-BNL Research Center, Brookhaven National Laboratory, Upton, NY 11973, USA,

Brookhaven National Laboratory, Upton, NY 11973, USA

E-mail: izubuchi@quark.phy.bnl.gov

We review the lattice QCD calculation of nuetron (and proton) electric dipole moment, and show the recent lattice calculation in dynamical fermion configurations.

Xth Quark Confinement and the Hadron Spectrum,

October 8-12, 2012

TUM Campus Garching, Munich, Germany

\footnotetext{
* Speaker.
} 


\section{Introduction}

Neutron electric dipole moment (EDM) is important observable for the search of not only QCD $\theta$-term in the Standard Model (SM) but also CP-violation beyond the SM (BSM). Neutron (and also proton) EDM has not been detected so far, and presently there is very tiny upper bound of neutron EDM $d_{N}<2.9 \times 10^{-26} \mathrm{e} \cdot \mathrm{fm}$ [1]. The $\mathrm{P}$ and CP-violating contribution via phase of CKM mass matrix is start from three-loop order in perturbation theory, and according to ref. [2, 3] CKM contribution has been estimated as $d_{N}^{\mathrm{CKM}} \simeq 10^{-30}-10^{-32} \mathrm{e} \cdot \mathrm{cm}$. which is 4-digit below the current experimental upper bound. While in the strong interaction there is $\mathrm{CP}$-violating $\theta$-term in QCD Lagrangian, tiny upper bound of EDM indicates that $\theta$-term may be strongly suppressed from unknown mechanism (strong $\mathrm{CP}$ problem). The simple solution is that one of the light quark mass $\left(m_{u, d}\right)$ vanishes due to cancellation between quantum correction of QCD and QED. However from lattice QCD calculation including QED correction into (valence) quark field we have known that $m_{u}=2.24(35)$ and $m_{d}=4.65(35)(\overline{M S}$ at $\mu=2 \mathrm{GeV})$ [4], and thus up-quark mass is finite over $7 \sigma$. It turns out that the zero quark mass solution of strong $\mathrm{CP}$ problem has been almost excluded. Although there have been several candidates of solution in low-energy region (axion model and one-loop calculation of $\theta$ ), the parameter region allowed from observation of cosmology and experiment has been narrow.

On the other hand for the BSMs, e.g. supersymmetry (SUSY) models, EDM of nucleon, atom and lepton are used to take a constraint on the parameter space of the new particle couplings and mass $[5,6,7,8,9]$ (also review of $[11,12]$ and reference therein). These are effectively expressed as the five-(or six-) dimension CP-violating bilinear operator with field strength of photon (quark EDM), gluon (chromo EDM) or purely gluon field (Weinberg operator), and there are several arguments of contribution to EDMs using some effective models [6, 9, 10]. Recently many experimental plans of neutron and proton EDM, dueteron EDM, atom EDM and lepton EDM are proposed $[13,15,14,16]$ to increase the presion. These experiments aim to take a strong bound toward $10^{-29} \mathrm{e} \cdot \mathrm{cm}$ sensitivity for neutron or proton EDM, and thus from theoretical side it will be neccessary to evaluate the precise contribution of hadronic effect for robustness on parameter space of BSMs. Lattice QCD plays an essential role.

So far there have been several attempts of the estimation of nucleon EDM from lattice QCD. In ref. $[18,19]$ they addressed that the strategy by inserting the external electric field on the lattice. In $[20,22]$ authors have elaborated two strategies of lattice calculation of neutron and proton EDM, and then [21,23] present the numerical results of nucleon EDM in $N_{f}=2$ QCD. Furthermore there are other attempts of lattice calculation of nucleon EDM with imaginary $\theta$ ensembles [24] or including higher dimensional CP-violating operator [25]. In this proceedings we show these strategies and some numerical results.

\section{External electric field method}

Regarding that nucleon EDM is defined as the energy-shift via interaction between nucleonspin direction and electric field, the Hamiltonian CP-violating in $\theta$ vacuum is described as

$$
\delta H_{\mathrm{CP}}=d_{N} \theta \vec{S} \cdot \vec{E}+\mathscr{O}\left(\theta E^{3}, E \theta^{3}\right)
$$


and thus the energy shift depending on the spin component is given in

$$
\mathscr{E}_{N+}^{\theta}-\mathscr{E}_{N-}^{\theta}=d_{N} \theta E_{z}
$$

with nucleon energy $\mathscr{E}_{N \pm}^{\theta}$ of spin $\pm 1 / 2$ in setting to $\vec{E}=\left(0,0, E_{z}\right)$ for external electric field.

Considering the Minkowski definition of z-direction external electric field, the transformation of link variable in quark propagator of flavor $q=u, d$ is

$$
U_{t}(x) \rightarrow e^{e_{q} E_{z} t} U_{t}(x) \equiv U_{t}^{q}(E, x), \quad U_{t}^{\dagger}(x) \rightarrow e^{-e_{q} E_{z} t} U_{t}^{\dagger}(x) \equiv\left(U_{t}^{q}\right)^{\dagger}(E, x),
$$

where we introduce quark charge $e_{q}$ for $e_{u}=2 / 3$ and $e_{d}=-1 / 3$ (in the case of using Euclidean electric field it is defined as pure imaginary value in the exponent). In ref.[22, 23] we employed the external electric field with valence quark. Under the assumption of SU(3) isospin symmetry, in which the summation of sea quark charge vanishes as $e_{u}+e_{d}+e_{s}=0$, we can ignore the isospin breaking of sea quarks up to the first order of expansion of electric field. Note that, since the Minkowski electric field has an introduction of violation of temporal periodicity i.e. $U_{t}^{q}(E, x+$ $\left.\hat{e}_{t} L_{t}\right) \neq U_{t}^{q}(E, x)$, in which there is large gap in the temporal boundary proportional to $e^{e_{q} E_{z} L_{t}}$, there exists enhanced contamination coming from temporal boundary.

In order to extract EDM from 2-point function of nucleon $\left\langle N_{s} \bar{N}_{s}\right\rangle_{\theta}(E)$ with each spin-component $s$ under electric field $E=(0,0, E)$ and $\theta$-term, it is useful to make the ratio of different spincomponent as,

$$
\begin{aligned}
& R_{3}(E, t ; \theta)=\frac{\left\langle N_{1} \bar{N}_{1}\right\rangle_{\theta}(E)}{\left\langle N_{2} \bar{N}_{2}\right\rangle_{\theta}(E)}, \\
& R^{\operatorname{corr}}(E, t ; \theta)=\frac{R_{3}(E, t ; \theta)}{R_{3}(-E, t ; \theta)} \frac{R_{3}(-E, t ; 0)}{R_{3}(E, t ; 0)} \simeq \frac{1+\theta A_{N}\left(E^{2}\right) E}{1-\theta A_{N}\left(E^{2}\right) E} \exp \left[-2 d_{N} E \theta t\right],
\end{aligned}
$$

in which the last equation is exact if we take the limit of large time separation. $\theta$-term is adopted by the reweighting form of $e^{i \theta Q}$ with topological charge $Q$. As a consequence EDM appears in the exponent proportional to electric field and $\theta$. Here the $E$ and $\theta$ are input parameters.

Figure 1 shows that $R_{3}$ as a function of time-slice of sink nucleon operator has the correct linear response to sign of electric field as expected in Eq.(2.5), and in the effective mass plot plateau region of $R_{\text {corr }}$ is considered as signal of EDM obtained by external electric field method.

\section{Parity and CP-odd form factor}

Considering the matrix element with the electromagnetic current between the nucleon state under $\theta$-vacuum, such matrix element can be expanded into the $\mathrm{P}$ and $\mathrm{CP}$-even form factor $F_{1,2}$ corresponding to electromagnetic moment, $\mathrm{P}$ and $\mathrm{CP}$-odd form factor $F_{3}$ corresponding to EDM and $\mathrm{P}$-odd form factor $F_{A}$ called as anapole form factor as

$$
\begin{aligned}
\left\langle N\left(\vec{p}_{1}, s\right)\left|J_{\mu}^{\mathrm{EM}}\right| N\left(\vec{p}_{0}, s\right)\right\rangle_{\theta} & =\bar{u}_{N}^{\theta}\left(p_{1}, s\right)\left[F_{1}\left(p^{2}\right) \gamma_{\mu}+F_{2}\left(p^{2}\right) \sigma_{\mu v} \frac{p_{v}}{2 m_{N}}\right. \\
& \left.+F_{3}^{\theta}\left(p^{2}\right) \gamma_{5} \sigma_{\mu v} \frac{p_{v}}{2 m_{N}}+F_{A}\left(p^{2}\right)\left(i p^{2} \gamma_{\mu} \gamma_{5}-2 m_{N} p_{\mu} \gamma_{5}\right)\right] u_{N}^{\theta}\left(\vec{p}_{0}, s\right)
\end{aligned}
$$



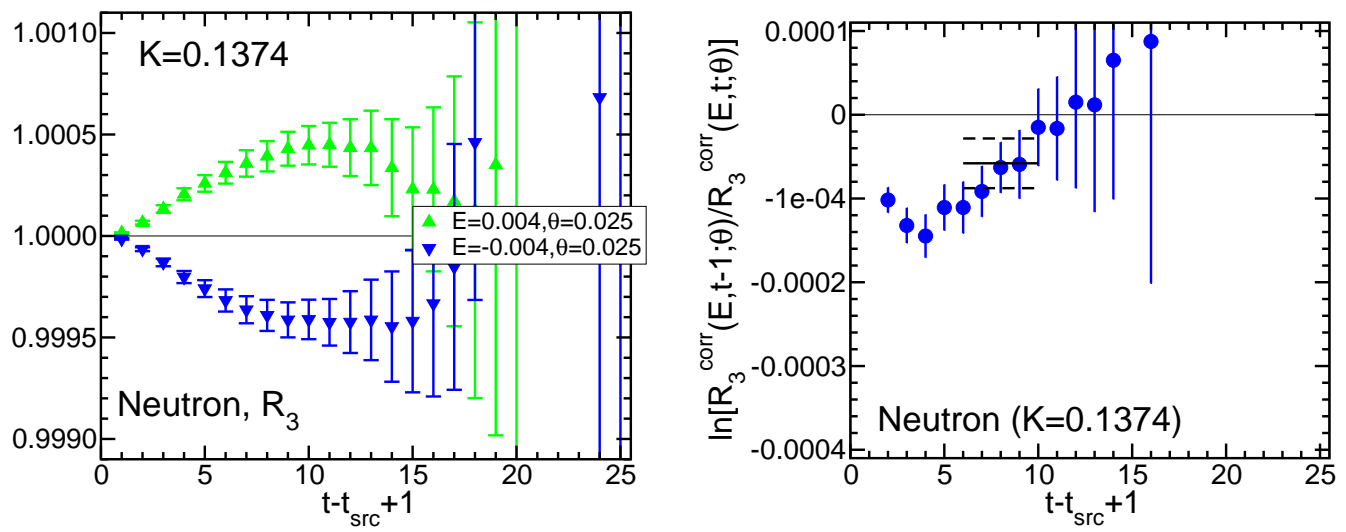

Figure 1: (Left) Time-dependence of $R_{\text {corr }}$ with positive (upper-triangle) and negative (down-triangle) electric field. (Right) The effective mass plot of the $R_{\text {corr }}$ The solid line denotes central value and dashed-line denotes the error band. I refer to ref.[23]. This is in $N_{f}=2$ clover fermion gauge configurations in CP-PACS collaboration [26]

where the Dirac spinor $u_{N}^{\theta}$ appearing in the above suffers the effect of $\theta$-term. Here the transfer momentum is defined as $p=p_{1}-p_{0}$. The on-shell Dirac spinor structure under $\theta$ vacuum is expressed as

$$
\sum_{s} u_{N}(\vec{p}, s) \bar{u}_{N}(\vec{p}, s)=\frac{i p \cdot \gamma+m_{N} e^{i \gamma_{5} \alpha_{N}(\theta)}}{2 E_{N}},
$$

from the argument in ref.[22] where $\alpha_{N}(\theta)$ is defined as the CP-odd phase whose leading term is $\alpha_{N}(\theta)=\alpha_{N} \theta+\mathscr{O}\left(\theta^{2}\right)$. In lattice QCD the form factor in Eq.(3.1) can be extracted from the three-point function formed as (nucleon)-(operator)-(nucleon); $\left\langle T\left\{\eta_{N} J_{\mu} \bar{\eta}_{N}\right\}\right\rangle_{\theta}$. Expanding up to the order of $\theta$, we have

$$
\left\langle T\left\{\eta_{N} J_{\mu} \bar{\eta}_{N}\right\}\right\rangle_{\theta}(t, \tau \mid \vec{p})=C_{J_{\mu}}(t, \tau \mid \vec{p})+i \theta C_{J_{\mu}}^{Q}(t, \tau \mid \vec{p})+\mathscr{O}\left(\theta^{2}\right),
$$

in which the second term includes EDM form factor $F_{3}$. As shown in ref.[22], the ratio of second term $C_{J_{\mu}}^{Q}(\vec{p}, t)$ and two-point function with smeared $C_{S}^{N}(t, \vec{p})$ and point $C_{P}^{N}(t, \vec{p})$ source,

$$
R_{\mu}^{Q}(t, \tau \mid p)=K \frac{C_{J_{\mu}}^{Q}(t, \tau \mid \vec{p})}{C_{S}^{N}(t, \vec{p})}\left[\frac{C_{S}^{N}(t, \vec{p}) C_{S}^{N}(\tau, \vec{p}) C_{L}^{N}(t-\tau, 0)}{C_{S}^{N}(t, 0) C_{S}^{N}(\tau, 0) C_{L}^{N}(t-\tau, \vec{p})}\right]^{1 / 2}
$$

with $K=\sqrt{2\left(E_{N}+m_{N}\right) / E_{N}}$ after taking the large time separation of $t-\tau$ and $\tau$ is written as its asymptotic form,

$$
\begin{aligned}
R_{J_{\mu}}^{\theta^{1}}\left(t_{1}, t, t_{0} \mid p_{1}, p_{0}\right) & \simeq \frac{\alpha_{N}}{2} \gamma_{5}\left[F_{1} \gamma_{\mu}+F_{2} \sigma_{\mu v} \frac{p_{v}}{2 m_{N}}\right] \frac{i p_{0} \cdot \gamma+m_{N}}{2 E_{N}} \\
& +\frac{1+\gamma_{4}}{2}\left[F_{1} \gamma_{\mu}+F_{2} \sigma_{\mu v} \frac{p_{v}}{2 m_{N}}\right] \frac{\alpha_{N} m_{N}}{2 E_{N}} \gamma_{5} \\
& +\frac{1+\gamma_{4}}{2}\left[F_{3} \gamma_{5} \sigma_{\mu v} \frac{p_{v}}{2 m_{N}}+F_{A}\left(i p^{2} \gamma_{\mu} \gamma_{5}-2 m_{N} p_{\mu} \gamma_{5}\right)\right] \frac{i p_{0} \cdot \gamma+m_{N}}{2 E_{N}} .
\end{aligned}
$$

From the above formula we know that the EDM form factor $F_{3}$ can be obtained by subtraction of CP-odd phase factor $\alpha_{N}$ times combination of CP-even form factor $F_{1,2}$ (the first and second 

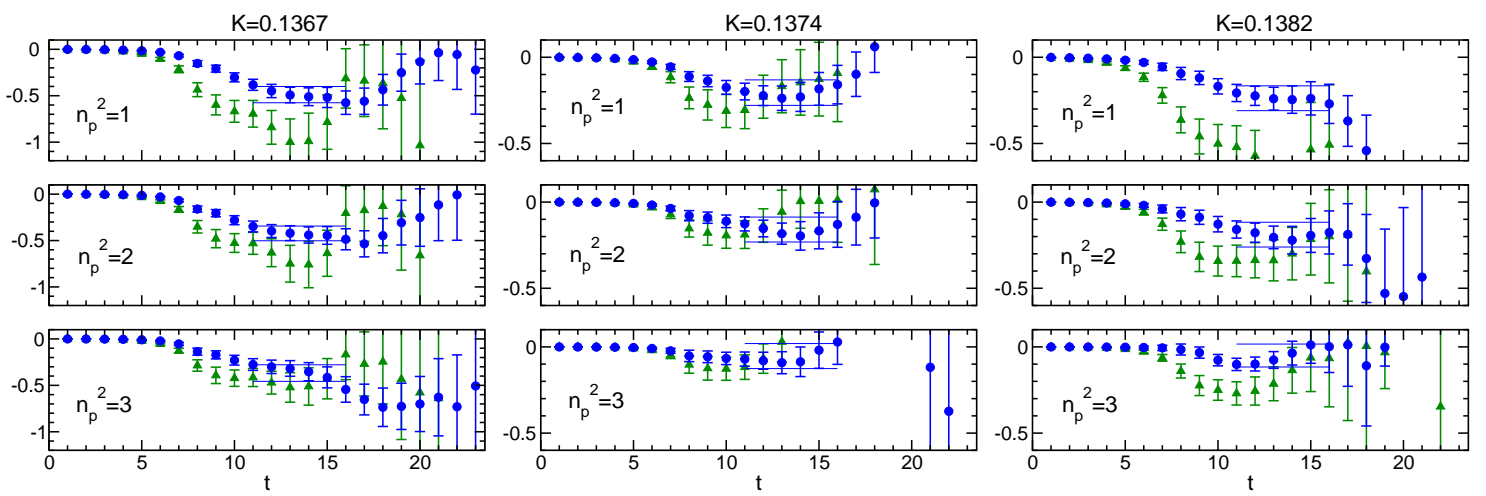

Figure 2: Three figures show the neutron EDM form factor at different time-slice of sink nucleon operator at Wilson mass parameter $K=0.1367$ (left), 0.1374(middle), 0.1382(right) with three lowest transfer momenta in $N_{f}=2$ clover fermion configurations [26]. These data correspond to $0.3-0.85 \mathrm{GeV}^{2}$ for pion mass squared. The different symbols denote the comparison of results with different sets of current direction (blue circle: $J_{t}$ and green triangle: $J_{z}$ ) and spin-projection matrix to check the consistency of the signal. The solid line shows the fitting result of blue circle symbols.

terms) which are evaluated from expansion of Dirac spinor $u_{N}^{\theta}$ of Eq.(3.2) and the leading order of three-point function $C_{J_{\mu}}(t, \tau \mid \vec{p})$ in Eq.(3.3).

Figure 2 shows the extracted EDM form factor following the formula in Eq.(3.5) (and also $[22,21]$ in details) from three-point function in $\theta$ term (here we also ignore the disconnected diagram in three-point function). These are data in which the source location of nucleon and operator location are fixed in $t=1$ and $t=8$ respectively. There appears the signal of neutron EDM between 11 and 16 for sink-time separation. Fitting the data in Figure 2, we obtain the transfer momentum dependence of neutron EDM form factor as shown in Figure 3. Since the neutron EDM is defined in the zero transfer momentum limit,

$$
d_{N}=\lim _{p^{2} \rightarrow 0} F_{3}\left(p^{2}\right) / 2 m_{N},
$$

we obtain this after taking the extrapolation to $p^{2}=0$ with linear fit function (Figure 3).

\section{Imaginary $\theta$}

Recently in ref.[27] they suggest that the new idea to avoid large statistical noise coming from reweighting with topological charge in $\theta$-term. Basically using analytic continuation into imaginary $\theta$ definition for $\theta$-term, the expectation value in QCD action $S_{\mathrm{QCD}}$ plus imaginary $\theta$ term action $\theta^{I} Q$ is represented as

$$
\langle O\rangle_{\theta^{I}}=Z_{\theta^{I}}^{-1} \int d U O[U] e^{S_{\mathrm{QCD}}[U]+\theta^{I} Q}, \quad Z_{\theta^{I}}=\int d U e^{S_{\mathrm{QCD}}[U]+\theta^{I} Q},
$$

and thus in the Monte-Carlo calculation EDM is given from real action $S_{\mathrm{QCD}}[U]+\theta^{I} Q$, which means there is no statistical noise due to sign problem, although it is necessary to carry out additional computation in the gauge ensembles including imaginary $\theta$ action. 


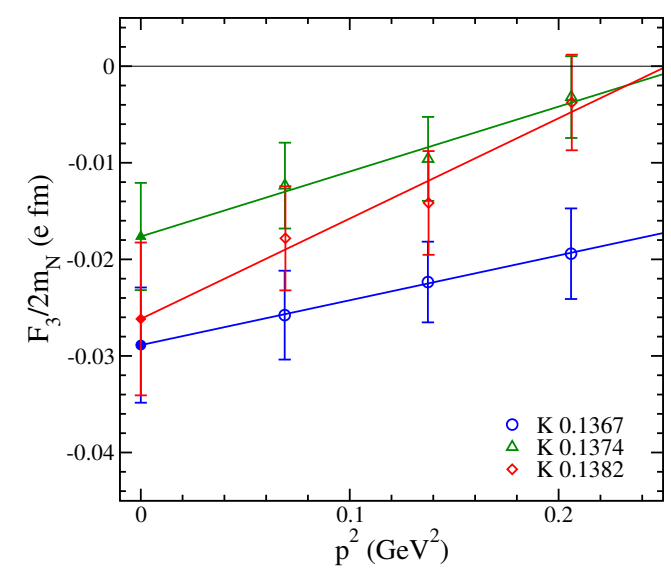

Figure 3: Neutron EDM form factor at three different transfer momentum squared obtained by fitting the data shown in Figure 2. The solid line shows the linear function fitting three transfer momenta $p^{2}$. The extrapolated data at $p^{2}=0$ is identified with the value of neutron EDM.

In ref.[24] they have applied this idea into measurement of neutron EDM form factor in relatively small lattice size using $N_{f}=2$ clover fermion. While they report the precise results of neutron and proton EDM about 10-20\% statistical accuracy, it may suffer huge systematic uncertainties due to breaking the chiral symmetry of valence clover fermion field connecting the anomaly term $\theta^{I} \bar{q} \gamma_{5} q$, which is related to lattice artifacts (see discussion in [19]).

\section{Summary and future plans}

Figure 4 plots the summary of lattice calculation with several lattice actions and methods for neutron and proton EDM in lattice QCD. We see that the two methods as shown in section 2 and 3 provide the consistent results for EDM within $1 \sigma$ error, whereas there is still large statistical error. Comparing between neutron and proton EDM in Figure 4, its flipped sign and similar magnitude of absolute value is in agreement with expectation from the argument of effective chiral perturbation theory [28, 29, 30] in which nucleon EDM is proportional to magnetic moment (and pion mass squared); magnetic moment of neutron (proton) is negative (positive) sign and its ratio is order one. Otherwise the neutron and proton EDM in Figure 4 seem to not depend on the pion mass squared in contrast with prediction of CP-symmetry in massless limit of QCD action. This may be due to remaining systematic error e.g. relatively heavy quark mass, finite volume effect $[31,32]$ and lattice artifacts.

Recently we carry out the lattice calculation in realistic lattice size $\left(2.7 \mathrm{fm}^{3}\right)$ with $N_{f}=2+1$ domain-wall fermion (DWF) configurations [33] at 300-400 MeV pion mass. This lattice calculation has much advantage to control the lattice artifacts of chiral symmetry breaking and enable us to improve the chiral behavior, whereas this computation is expensive. The main task to obtain more precise value of nucleon EDM in this ensemble is that we need to significantly accumulate statistics in Monte-Carlo simulation with the more efficient way, and hence we recently develop the numerical algorithm (AMA algorithm [34]) which is able to reduce the statistical error of correlator 

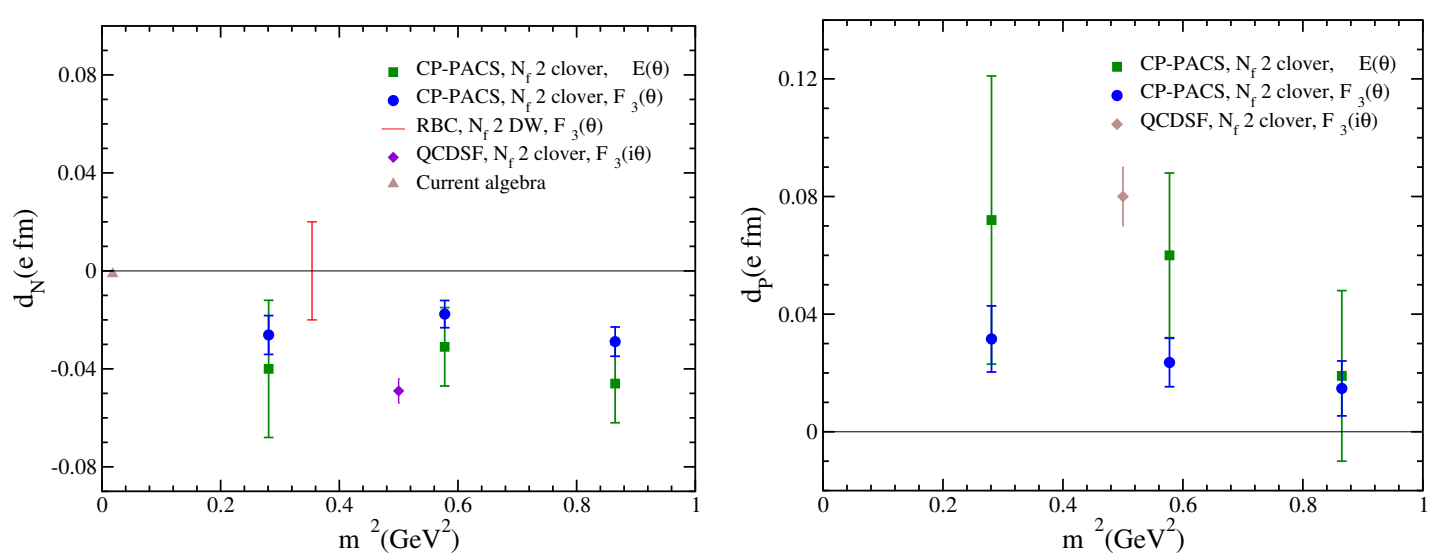

Figure 4: The summary plot of neutron EDM (left) and proton EDM (right) at several pion mass squared. The green-square symbols are results in external electric field method in $N_{f}=2$ clover fermion configurations quoted in [23], and blue-circle symbols are results obtained by EDM form factor after extrapolation toward physical kinematics in the same configurations as green-square one. The red bar denotes the error band estimated in [21] in $N_{f}=2$ domain-wall fermion configurations from EDM form factor. The diamond is a result from EDM form factor in imaginary $\theta$ ensemble of $N_{f}=2$ clover fermion configurations quoted in [27]. These errors are only statistical one. As noticed in section 4, the data of diamond may hide a large systematic uncertainty of chiral symmetry breaking of valence clover fermion field. We show an estimate in current algebra [28] (triangle) in the physical point for the reference (also see [30] in which they discussed about validity of this estimate).

evaluated in Monte-Carlo simulation by factor 5-10. This is much useful to persue the precise study for the observables evaluated in Monte-Carlo simulation, especially for nucleon EDM calculation.

This work was supported by the Japanese Ministry of Education Grant-in-Aid, Nos. 22540301 (TI), 23105714 (ES), 23105715 (TI) and U.S. DOE grants DE-AC02-98CH10886 (TI) and DEFG02-92ER40716 (TB). We also thank BNL, the RIKEN BNL Research Center, the RICC at RIKEN and USQCD for providing resources necessary for completion of this work.

\section{References}

[1] C. A. Baker, D. D. Doyle, P. Geltenbort, K. Green, M. G. D. van der Grinten, P. G. Harris, P. Iaydjiev and S. N. Ivanov et al., Phys. Rev. Lett. 97, 131801 (2006) [hep-ex/0602020].

[2] I. B. Khriplovich and A. R. Zhitnitsky, Phys. Lett. B 109, 490 (1982).

[3] A. Czarnecki and B. Krause, Phys. Rev. Lett. 78, 4339 (1997).

[4] T. Blum, R. Zhou, T. Doi, M. Hayakawa, T. Izubuchi, S. Uno and N. Yamada, Phys. Rev. D 82, 094508 (2010) [arXiv:1006.1311 [hep-lat]].

[5] S. Abel, S. Khalil and O. Lebedev, Nucl. Phys. B 606, 151 (2001) [hep-ph/0103320].

[6] J. Hisano and Y. Shimizu, Phys. Rev. D 70, 093001 (2004) [hep-ph/0406091].

[7] J. R. Ellis, J. S. Lee and A. Pilaftsis, JHEP 0810, 049 (2008) [arXiv:0808.1819 [hep-ph]].

[8] Y. Li, S. Profumo and M. Ramsey-Musolf, JHEP 1008, 062 (2010) [arXiv:1006.1440 [hep-ph]]. 
[9] J. Ellis, J. S. Lee and A. Pilaftsis, JHEP 1102, 045 (2011) [arXiv:1101.3529 [hep-ph]].

[10] J. Hisano, J. Y. Lee, N. Nagata and Y. Shimizu, Phys. Rev. D 85, 114044 (2012) [arXiv:1204.2653 [hep-ph]].

[11] T. Ibrahim and P. Nath, Rev. Mod. Phys. 80, 577 (2008) [arXiv:0705.2008 [hep-ph]].

[12] T. Fukuyama, Int. J. Mod. Phys. A 27, 1230015 (2012).

[13] Dave Wark, J. Phys. Conf. Ser. 171, 012002 (2009).

[14] Yannis K Semertzidis, J. Phys. Conf. Ser. 335, 012012 (2011).

[15] Hiromi Iinuma for J-PARC New g-2/EDM experiment collaboration, J. Phys. Conf. Ser. 295, 012032 (2011).

[16] O. Bourrion, G. Pignol, D. Rebreyend and C. Vescovi, Nucl. Instrum. Meth. A 701, 278 (2013) [arXiv:1207.0618 [physics.ins-det]].

[17] M. Pospelov and A. Ritz, Annals Phys. 318, 119 (2005).

[18] S. Aoki and A. Gocksch, Phys. Rev. Lett. 63, 1125 (1989) [Erratum-ibid. 65, 1172 (1990)].

[19] S. Aoki, A. Gocksch, A. V. Manohar and S. R. Sharpe, Phys. Rev. Lett. 65, 1092 (1990).

[20] E. Shintani, et al., Phys. Rev. D 72, 014504 (2005).

[21] F. Berruto, T. Blum, K. Orginos and A. Soni, Phys. Rev. D 73, 054509 (2006).

[22] E. Shintani, et al., Phys. Rev. D 75, 034507 (2007).

[23] E. Shintani, S. Aoki and Y. Kuramashi, Phys. Rev. D 78, 014503 (2008).

[24] R. Horsley, T. Izubuchi, Y. Nakamura, D. Pleiter, P. E. L. Rakow, G. Schierholz and J. Zanotti, arXiv:0808.1428 [hep-lat].

[25] T. Bhattacharya, V. Cirigliano and R. Gupta, PoS LATTICE 2012, 179 (2012) [arXiv:1212.4918 [hep-lat]].

[26] A. Ali Khan et al. [CP-PACS Collaboration], Phys. Rev. D 65, 054505 (2002) [Erratum-ibid. D 67, 059901 (2003)] [hep-lat/0105015].

[27] T. Izubuchi, S. Aoki, K. Hashimoto, Y. Nakamura, T. Sekido and G. Schierholz, PoS LAT 2007, 106 (2007) [arXiv:0802.1470 [hep-lat]].

[28] R. J. Crewther, P. Di Vecchia, G. Veneziano and E. Witten, Phys. Lett. B 88, 123 (1979) [Erratum-ibid. B 91, 487 (1980)].

[29] K. Kawarabayashi and N. Ohta, Nucl. Phys. B 175, 477 (1980).

[30] S. Aoki and T. Hatsuda, Phys. Rev. D 45, 2427 (1992).

[31] D. O'Connell and M. J. Savage, Phys. Lett. B 633, 319 (2006) [hep-lat/0508009].

[32] F. -K. Guo and U. -G. Meissner, JHEP 1212, 097 (2012) [arXiv:1210.5887 [hep-ph]].

[33] Y. Aoki et al. [RBC and UKQCD Collaborations], Phys. Rev. D 83, 074508 (2011).

[34] T. Blum, T. Izubuchi and E. Shintani, arXiv:1208.4349 [hep-lat]; T. Blum, T. Izubuchi and E. Shintani, PoS LATTICE 2012, 262 (2012) [arXiv:1212.5542 [hep-lat]]. 